\title{
Design of Intelligent Customer Service Questioning and Answering a System for Power Business Scenario Based on AI Technology
}

\author{
Shisong Wu \\ Digital Grid Research Institute, CSG, Guangzhou, China \\ Correspondence should be addressed to Shisong Wu; wushisong@yccxip.com
}

Received 29 October 2021; Accepted 27 December 2021; Published 27 January 2022

Academic Editor: Jinyan Song

Copyright ( 2022 Shisong Wu. This is an open access article distributed under the Creative Commons Attribution License, which permits unrestricted use, distribution, and reproduction in any medium, provided the original work is properly cited.

\begin{abstract}
In order to solve the problems of large demand for power business and small number of customer service, an intelligent customer service questioning and answering a system for power business scenario based on AI technology is designed. The approach first uses the particle swarm optimization algorithm to automatically classify the question attributes and then uses the fuzzy $c$-means clustering algorithm to match the answers with the highest similarity to the questions and return to the customers. The system collects the questions raised by customers through the acquisition module, uploads the question work order to the knowledge base through the information assistance module, and stores the preprocessed questions to the knowledge base. After completing the problem attribute classification through the particle swarm clustering algorithm classification model in the batch analysis and calculation module, the question answers are matched through fuzzy $c$-means clustering. At the same time, the similarity of different keywords is calculated to find a series of related questions. After the obtained data are analyzed in real time through the self-service customer service module, the answer is extracted and fed back to the customer, and the question answer is presented to the customer in the system interface. The experimental results show that the designed system has low worst-case time complexity, which is up to 0.35 only. The reason is that the system in this paper can use priority information to deal with the problems raised by customers, which is different from the past work where dealing with customers request via priority information is not used. The system can give the corresponding answers according to the customer's options. It has convenient operation, high integrated control ability, and good information management performance. Compared with the traditional approach which could waste a lot of resource and data, the proposed approach can reduce the differences between problem data, eliminate invalid data, and simplify the data classification process. The application of the system can effectively accelerate the information transmission efficiency of the power company and can be used for power exchange platform automation in the future.
\end{abstract}

\section{Introduction}

'The related businesses of power companies in different scenarios are gradually increasing, and some businesses have changed from offline services to various online remote services $[1,2]$. However, both offline service and remote service will waste power business resources [3], such as the shortage of customer service personnel and the difference of business capacity [4], which will directly affect the service level. Therefore, the design of a high-quality power business intelligent customer service system has attracted the attention of relevant scholars [5].
Yt et al. [6] propose a method to construct the knowledge graph of power customer service domain and constructed a knowledge graph containing more than 15,000 entities and more than 20,000 relationships. Based on the knowledge map, an intelligent question answering application architecture is designed. The architecture is composed of several functional modules, such as dialogue process configuration, natural language processing, and business process processing. Provide more efficient and open knowledge retrieval services for power customer service business and improve the intelligent level of customer service questions and answers. However, this method has little experience in 
customer service operation and maintenance and cannot use knowledge sharing to deal with problems raised by customers, resulting in massive historical operation and maintenance data being wasted. Li et al. [7] point out that customer service is changing from traditional manual service to automatic service. Automatic service uses different computational informatics to achieve more efficient and high-quality service. Automated customer service requires expertise in big data and data analysis as a prerequisite. However, many companies, especially small and mediumsized enterprises, do not have enough data and experience due to limited scale and resources. They need to rely on third parties, resulting in the lack of development of core customer service capabilities. In order to overcome these challenges, it proposes an open automatic customer service platform based on the Internet of things (IoT), blockchain, and automatic machine learning (AUTOML). Yu et al. [8] analyze the changes of the new generation power grid dispatching control system in system architecture, humancomputer interaction mode, business organization mode, and so on. This paper combs the new requirements of the new generation power grid dispatching control system for access control and puts forward the service access control solution in the new generation power grid dispatching control system. The key technologies such as path-based resource identification definition, metadata-based resource management, rule-engine-based multifactor access control, and cross-system access control based on parent-child organization relationship are studied. The scheme has been verified in the prototype system and provides a multidimensional security access control means for the business in the new-generation power grid dispatching control system. However, during the time period when the system has no customer service, customers need to wait for the customer service to go to work.

Based on the aforementioned analysis, although most of the existing methods have realized the intelligent question answering of power business, there are still some problems such as poor keyword searching, inability in keywords decomposition of most customers, low data reusing rate, poor efficiency in question answering content management, and weak pertinence of answers $[9,10]$. Therefore, we choose to apply the fuzzy $c$-means clustering algorithm [11], which can directly deal with the problems that a text belongs to multiple text classes, and class boundaries are fuzzy and overlapping regardless of the clustering category. Based on the aforementioned justification, this paper proposes to build an intelligent customer service questioning and answering a system for power business scenarios based on AI technology. AI technology has been widely used in the area of prediction [12], state estimation [13, 14], control [15-17], and optimization [18] and has made considerable contribution. Therefore, this research tries AI technology. The approach first uses the particle swarm optimization algorithm to automatically classify the question attributes and then uses the fuzzy $c$-means clustering algorithm to match the answers with the highest similarity to the questions and return to the customers. The approach can reduce the differences between problem data, eliminate invalid data, and simplify the data classification process. The system in this paper can use priority information to deal with the problems raised by customers, so it has a low worst-case time complexity.

\section{Power Business Scenario Intelligent Customer Service Questioning and Answering a System Based on AI Technology}

2.1. Overall System Structure. In order to improve the customer service quality of power business, an intelligent customer service questioning and answering a system for power business scenario based on AI technology is constructed. As shown in Figure 1, the problems raised by customers are solved by the designed system to improve the customer service quality.

As can be seen from Figure 1, the system collects various questions raised by customers through the acquisition module and then transmits the questions to the knowledge base in the form of work order through the auxiliary information module to realize the word, marking and identification of the preprocessed questions, and store them in the knowledge base. The answers matching with the answers with the highest similarity to the work order are retrieved in the batch analysis and calculation module. After the data are analyzed in real time by the self-service customer service module, the answers are extracted. The core module of the system is the batch analysis and calculation module, which uses the particle clustering algorithm to divide the attributes of the questions raised by customers and then uses the fuzzy $c$-means clustering algorithm to match the keywords related to the topic in the text, calculate the similarity of different keywords, find a series of related questions, and finally get the answers with the highest matching degree. The fuzzy $c$-means clustering algorithm is used to match the keywords related to the topic in the text, calculate the similarity of different keywords, find the answers related to relevant questions, and finally feed back to customers through the language processing of independent customer service to realize interactive questions and answers A with customers.

2.2. Problem Acquisition Module. The main function of this module is to collect relevant questions raised by users, count and divide them into a specific server directory, and convert them into intelligent customer service questions and answers A data of power business scenario. The main work of this module includes the following:

(1) Determine the time interval for the system to collect problem data, which is determined as $3 \mathrm{~min}$.

(2) Apply TXT file type to store relevant files with problems in the system.

(3) Define the coding format of system acquisition problem file as UTF-8, build a data interface in series with Hadoop file system, obtain the problem, and save it in Hadoop. 


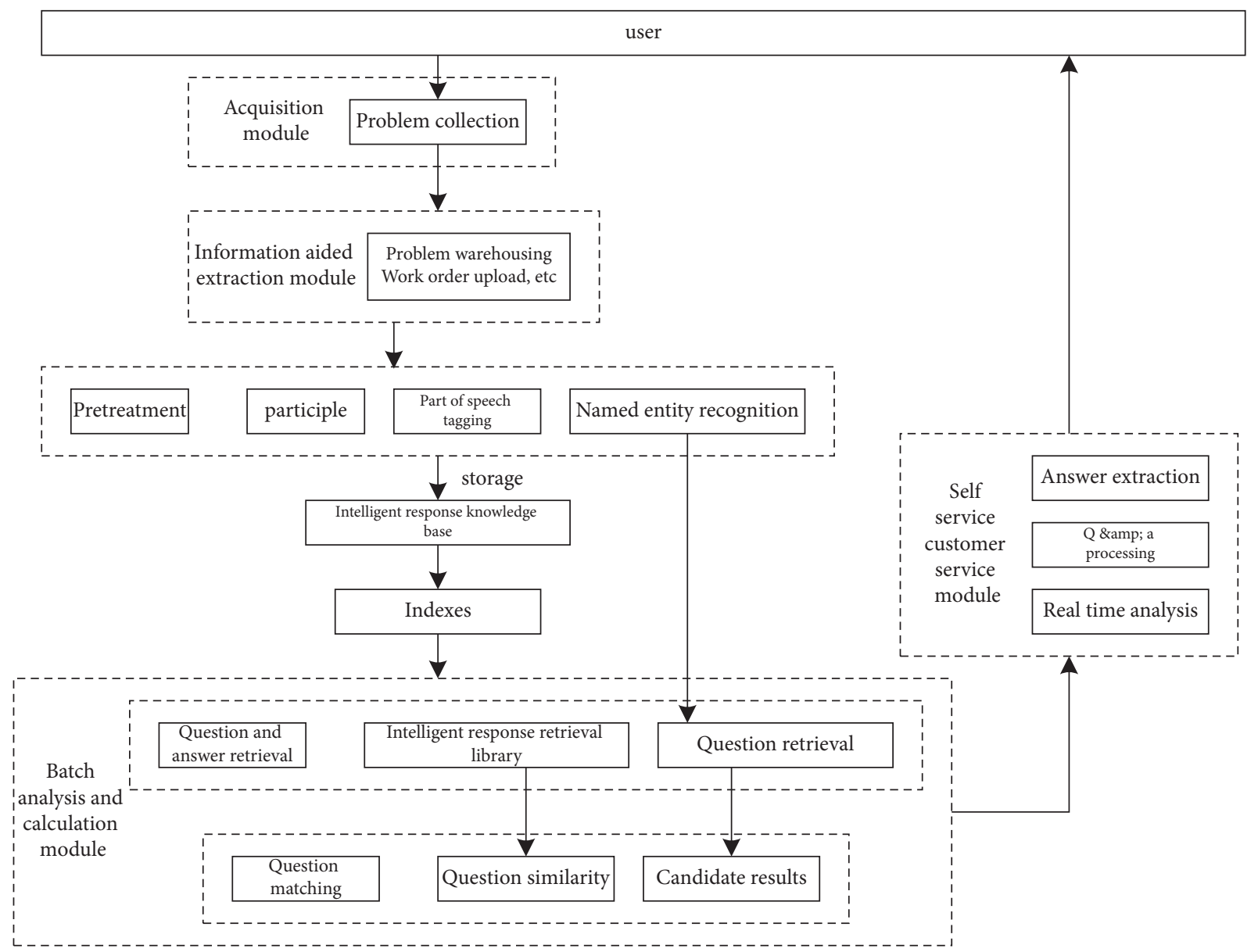

FIgURE 1: Power business scenario intelligent customer service questioning and answering a system.

2.3. Auxiliary Information Extraction Module. Figure 2 shows the details of the information-aided extraction module.

The four main functions of the information-aided extraction module are as follows: problem warehousing, transmitting work orders, changing problems to the knowledge base, and manually modifying the content of the knowledge base to the response base. As seen from Figure 2, if the customer raises a question, the module will automatically reply to the customer, submit the unsolvable problem to the service queue, provide various feedback methods to the response base, and then answer automatically through the knowledge base.

\subsection{Batch Analysis and Calculation Module}

2.4.1. Problem Attribute Classification. The core function of this module is to deeply analyze the collected operation problems and calculate the system. This method takes the existing problem data as the comparison basis to verify the similarity and clustering relationship between the problems. As the traditional word segmentation will produce a large number of resource waste and inefficient problems when putting forward the questions to be answered to the system, the module first uses the particle swarm optimization algorithm to automatically classify the question attributes $[19,20]$ and finally uses the fuzzy $c$-means clustering algorithm to match the answers with the highest similarity to the questions and return to the customers. Figure 3 shows the problem classification process.

In Figure 3, the general flow of problem attribute classification is described, which is, the preprocessed data set is pretrained. In the embedding layer, according to the word vector strategy, particle swarm clustering algorithm is used to complete the transformation of sentence vector, and a persistent classification model, which meets the standard accuracy and can be used for future classification is obtained through training.

\subsubsection{Fuzzy c-Means Clustering Mathematical Model.} Fuzzy $c$-means clustering algorithm is used for classification according to the similarity of the problem, and the ones with high similarity are judged as similar ones and classified into one group. Fuzzy $c$-means clustering is an unsupervised learning problem $[21,22]$. For the problem data with unclear classification, the strategy can automatically match the best keywords in the text after training. Using fuzzy $c$ means clustering algorithm can improve the compactness of clustering data and change the dispersion between clusters. 


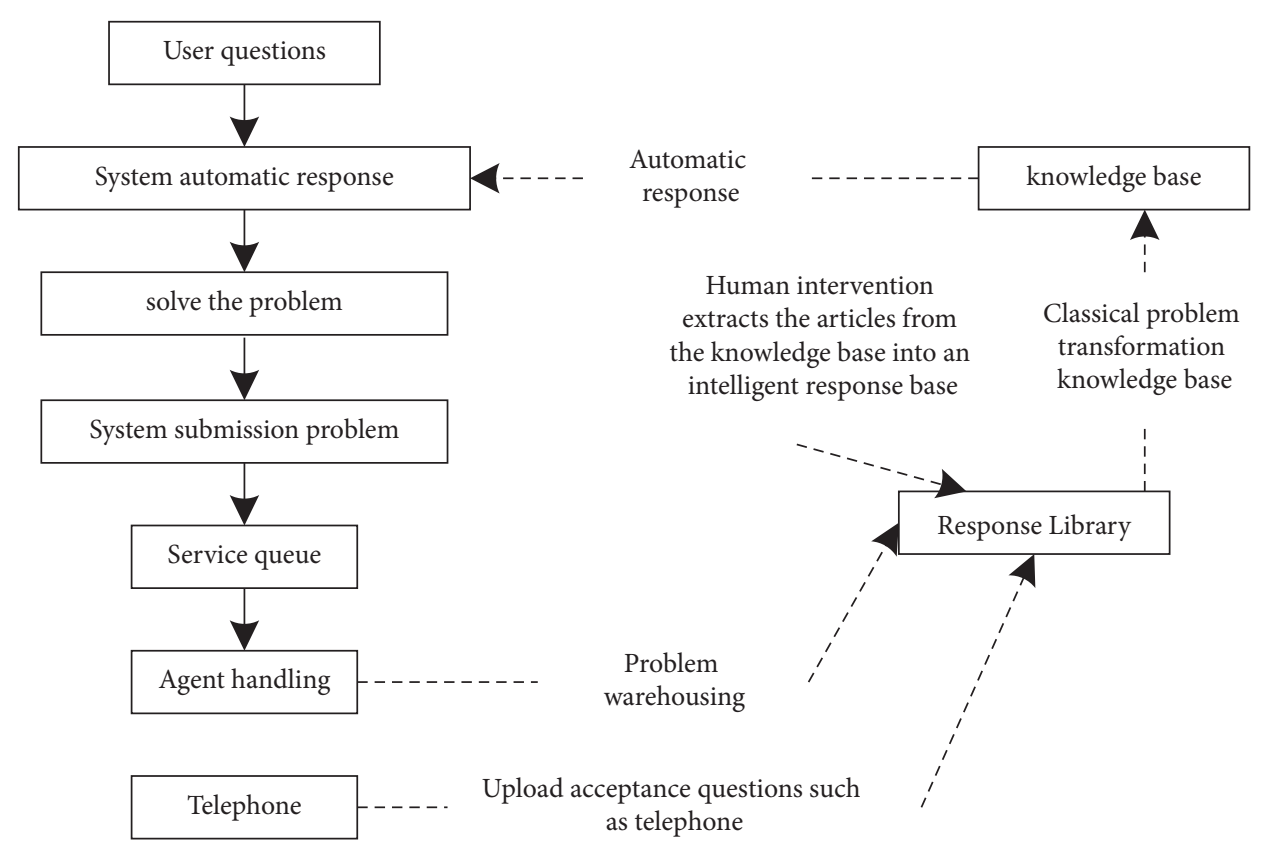

FIGURE 2: Auxiliary information-aided extraction module.

The fuzzy $c$-means clustering algorithm includes the following steps:

Step 1: Import the determined clustering parameter, $e$, and the sample set of the data object.

Step 2: Arbitrarily select sample points of $e$ and record them as the original cluster center $T_{1}(a), T_{2}(a)$, $\ldots, T_{e}(a)$, indicating the number of iterations. First, the iteration number $a=1$.

Step 3: Based on the minimum distance specification, the samples are divided into class $e T_{j}(e)$ represented by the cluster center with the minimum distance, and the number of samples in each class is $a_{j}(e)$. The minimum distance specification is calculated as follows:

$$
d=\sum_{i=1}^{a} \min _{j \in(1,2, \ldots e)}\left\|x_{i}-T_{j}\right\|^{2} .
$$

Step 4: After calculating the center of gravity of each category, take it as a new cluster center to obtain the following equation:

$$
T_{j}(e+1)=\frac{1}{a_{j}(k)} \sum_{X_{i} \in T_{j}(e)}^{a} x_{i},
$$

where, $j=1,2, \ldots, e, N_{j}(e)$ is the number of samples in $T_{j}(e)$, and the fuzzy $c$-means clustering algorithm needs to obtain the mean of $e$ new clustering centers.

Step 5: Perform secondary division and iteration according to the new cluster center and terminate the iteration when the sample is consistent with the criterion function of distance. follows:

$$
D_{j}=\sum_{j=1}^{e} \sum_{i=1}^{a}\left\|x_{i}-T_{j}\right\|^{2} .
$$

Formula (3) can effectively reduce the differences between problem data, eliminate invalid data, and simplify the data classification process. The fuzzy $c$-means clustering algorithm is used to normalize the order of magnitude of different problems so that it can uniformly expand the problem attribute classification on the order of magnitude and ensure the accuracy of the results.

2.5. Self-Service Customer Service Module. The self-service customer service module based on Web browser displays the intelligent analysis results of system operation. The selfservice customer service module realizes the functions of real-time analysis, answer processing, and answer extraction. It can match the logic and similarity of questions in the batch analysis and calculation module, extract high-precision answers through the answer extraction function, and give feedback to customers on the online customer service page.

\section{Experimental Analysis}

This experiment takes iris data set and wine data set as the research object to test the fuzzy $c$-means clustering algorithm applied in the system. The clustering effect is shown in Figure 4.

As can be seen from Figure 4, the system in this paper can get good clustering effect after four cycles. The clustering points are clearer and clearer, and the clustering points are highly dense. There is no wrong clustering, indicating that the system in this paper has strong clustering ability. 


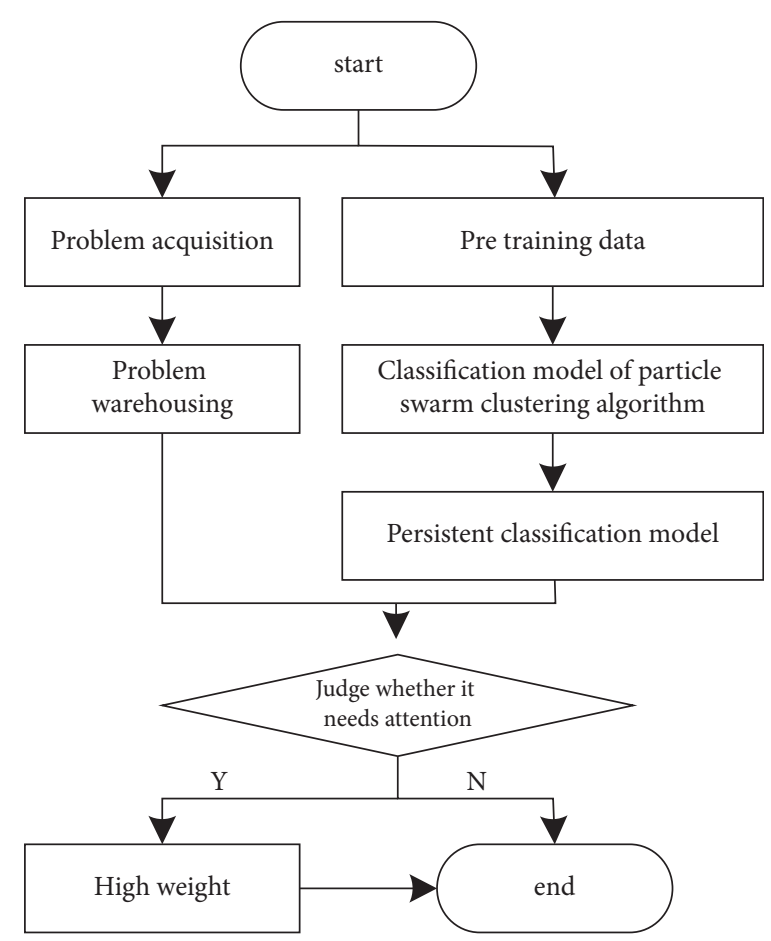

Figure 3: Problem attribute classification process.

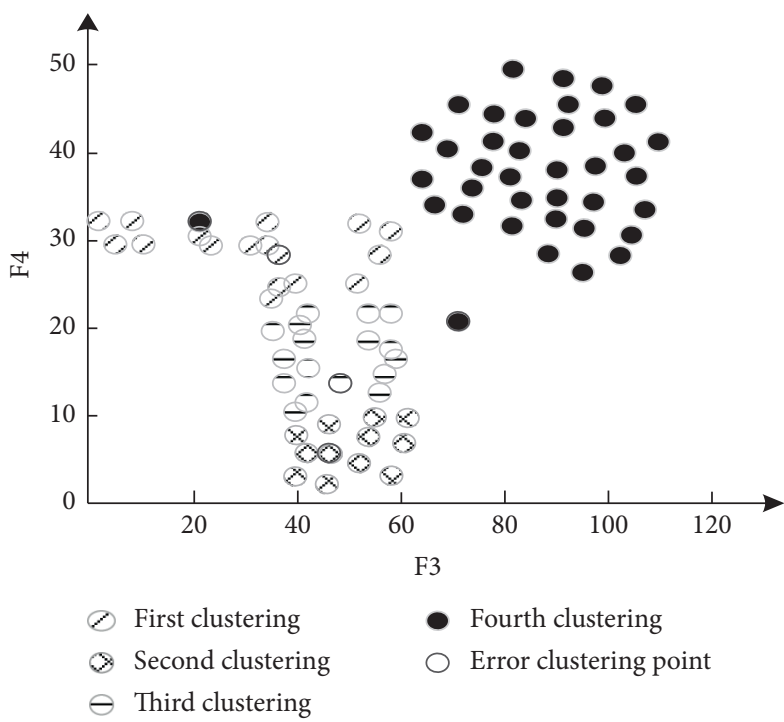

Figure 4: System clustering effect of this paper using fuzzy $c$-mean clustering method.

The designed system is compared with literature [6-8]. After 25 experiments on iris and wine data sets, the comparison results of the accuracy of the four systems are shown in Table 1.

It can be seen from Table 1 that the fuzzy $c$-means clustering algorithm adopted by the system in this paper can make the system obtain better clustering effect. Compared with the other three systems, the accuracy of the system application strategy in this paper is the highest in both data sets. In the process of experiment, this method can save storage space and reduce system running time.
3.1. Application Scenarios. In the experiment, taking intelligent speech technology as the core, the application scenarios such as voiceprint recognition, voice input method, automatic map adjustment, status query, question answering, page switching, and voice ticket issuing are designed. Voiceprint recognition and voice input method can be used as services to support other applications.

3.1.1. Voiceprint Recognition. The dual-recognition technology of voiceprint and voice is used for identity 
TABLE 1: Cluster comparison results between the research and past work.

\begin{tabular}{lcc}
\hline Algorithm & Iris dataset (\%) & Wine dataset (\%) \\
\hline Design system & 97.25 & 98.33 \\
Yt et al. study [6] & 88.38 & 93.31 \\
Li et al. study [7] & 83.57 & 95.27 \\
Yu et al. study [8] & 95.61 & 97.64 \\
\hline
\end{tabular}

authentication to improve the recognition accuracy of login customers. Subsequent operations are bound with login customers to automatically record the working status, behavior, and habits of each regulator, so that the intelligent assistant can provide services more effectively. At the same time, voiceprint recognition service can be provided for other applications as an option for authentication.

Through voiceprint, the customer can log in quickly to avoid the cumbersome operation of entering password. The system automatically records the login status and associates the operations in the next period with the logged in customer.

Example: Voiceprint login.

Input voice: The controller inputs "XXX of a city dispatching" through mic.

Recognition confirmation: The background program recognizes the voiceprint information and returns the name of the controller.

Login: The controller checks the recognized name on the interface. If it is correct, click login directly. If it is incorrect, select it from the drop-down menu, and then click login.

Operation confirmation: Before the control personnel carry out important operations, for example, confirming the operation ticket, they can verify their identity by voiceprint and can only be carried out after confirmation.

3.1.2. Voice Input Method. Text input through voice can replace the function of the existing input method. The input text can act on any standard text input control such as word, web page input box, and OMS page to realize convenient text input. Note: this function is limited to Windows system. An example of documentation is shown as follows:

Step 1: Open word and start the voice input method function.

Step 2: Speak the text content to be entered normally through mic, and the recognized text will be displayed on the word page in real time.

Step 3: Further editing and improvement can be made by customers.

Repeat Steps 2 and 3 until documentation is complete.

3.1.3. Automatic Drawing Adjustment. In the $\mathrm{D} 5000$ graphic environment, the designated system diagram or plant station diagram is automatically opened by the voice of the controller to achieve the effect of rapid page switching. At the same time, it supports the positioning function of the designated equipment and improves the work efficiency of the controller. At this time, the control robot interface remains suspended.

Example: "please open the diagram of landing station" or "read the diagram of landing station."

Keywords "open," "plant and station map," "access."

Parameter: [Landing station].

Effect: open the specified station diagram.

3.1.4. Status Query. According to the voice requirements of the regulator, automatically collect relevant information from the regulation cloud or other systems, query the current or historical status of the power grid, broadcast in the form of voice and text, and visually display through customized templates.

Example: "please query the total power supply of an area on April 17."

Keywords "query" and "total power supply".

Parameters: [April 17], [a region].

Effect: Query the total power supply on the specified date from the control cloud and broadcast through voice.

3.1.5. Problem Solving. The city dispatching knowledge base based on offline modeling realizes the intelligent question and answer function between man and machine, supports the ability of multiround dialogue, and supports the online update of knowledge base. The content of knowledge base can include basic information of municipal dispatching, regulation regulations, power system knowledge, and so on. At the same time, it provides knowledge base management tools to supplement and update knowledge.

Example 1: "what are the local dispatching equipment?"

Voice broadcasting: Power plants, substations, lines, and other primary and secondary equipment for mode adjustment and switching operation ordered by the municipal dispatching. Text display: (1) machines and boilers of all power plants with $110 \mathrm{kV}$ and above connected to a regional power grid, primary and secondary equipment of booster station and their parallel network cables; (2) $220 \mathrm{kV}$ bus and switchgear of $500 \mathrm{kV}$ substation; (3) $220 \mathrm{kV}$ ring network equipment; (4) $110 \mathrm{kV}$ and above load lines and corresponding side intervals; (5) substation equipment of $110 \mathrm{kV}$ and above (excluding local dispatching equipment); (6) power grid equipment assigned by superior dispatching 
to municipal dispatching; and (7) other equipment that the city dispatching deems necessary for direct dispatching.

3.1.6. Vice Ticket Issuing. The operation ticket can be processed by voice, including browsing the previous ticket and querying the current ticket status. For the new operation ticket, the text information can be entered by voice.

Example: "view the operation ticket being executed."

Keywords "view," "operation ticket."

Parameter: [executing].

Effect: Retrieve the operation tickets in circulation and display them in a list.

\subsection{Power Business Problem Attribute Classification Effect.} Through the analysis of the aforementioned application scenarios, it can be seen that the problem information of power companies is large and diverse. Effective classification can enhance the management effect of the system to a certain extent. For the test of system classification performance, five types of information such as power outage information, customer information, payment information, and meter reading time of power companies are classified and tested. These five types of information come from different communities and floors respectively. The classification performance of the system is measured by the attribute category of the problem, and the results are shown in Figure 5.

According to the test results in Figure 5, the system in this paper can effectively classify the attributes of five kinds of information. It can be seen that the payment information is close to the payment time attribute, indicating that the system in this paper can realize information classification according to the attributes of the problem and has a good classification effect.

3.3. Questions and Answers a Processing Effect. In order to deeply analyze the problem processing effect of the system, the posterior probability value of all data is used to measure the worst time complexity. The formula is as follows:

$$
\left(\tau_{1}+m \tau_{2}\right) \cdot n \cdot k=n k \tau_{1}+m n \tau_{2} .
$$

The number of information, attributes, and categories are described by $n, m$, and $k$, respectively; the posterior probability of certain information and the time of information increase are calculated by $\tau_{1}$ and $\tau_{2}$, respectively. For the case where $n, m$, and $k$ are the same, the calculation formula of the worst time complexity is as follows:

$$
O\left(n^{3}\right)=n^{2} \tau_{1}+n^{3} \tau_{2}
$$

The worst time complexity is set as 0.5 , the limit value to test the worst time complexity of the system intelligent questions and answers $\mathrm{A}$ in this paper. The worst time complexity of the system intelligent questions and answers A is negatively correlated with the system problem processing performance. The worst time complexity of the system intelligent questions and answers $\mathrm{A}$ is shown in Figure 6.

As can be seen from Figure 6, although the number of keywords is increasing, the worst-case time complexity of the system in this paper has increased. However, the increase is small, stable, and always lower than the limit value. This is because the system in this paper can use priority information to deal with the problems raised by customers, so it has a low worst-case time complexity. When the number of keywords is 25 , the worst time complexity is 0.35 , which shows that the system has good information management performance.

3.4. Effect of Power Business Resource Scheduling and Registration. The power business resource scheduling attenuation and registration degree of the design system are shown in Figures 7 and 8.

As can be seen from Figure 7, the information transmission performance and anti-inter symbol crosstalk performance of the system in this paper have been significantly improved. But they are lower than $4 \mathrm{~dB}$, indicating that the information transmission performance of the system in this paper is good, has good anti-inter symbol crosstalk performance, and can optimize the information management performance of the system.

As shown in Figure 8, in the case of resource mobilization, the system achieves $88 \%$ regardless of resource size and registration degree. The increase of resource size has little impact on registration. When the resource size is $40 \mathrm{~GB}$, the registration degree is the highest, reaching $97.8 \%$, and when the resource size is $100 \mathrm{~GB}$, the registration degree is $95.4 \%$, indicating that the system has good information management effect. It can correctly realize resource matching and scheduling.

3.5. Work Efficiency. In order to verify the application effect of the system in the actual operation process, the work efficiency of the power company before and 1 month after the application of the system is compared. The results are shown in Figure 9.

As can be seen from Figure 9, after the application of this system, the processing time of customers' questions is significantly reduced, and customers can get corresponding answers in a short time. Before the application of this system, the processing time of customers' questions was longer because the number of customers is limited, customers need to wait in line, wasting more time, and the efficiency of power companies serving customers is low The problems raised by customers after the application of the system greatly improve the operation efficiency of the power company and the work efficiency of the operation and maintenance group of the power company. 


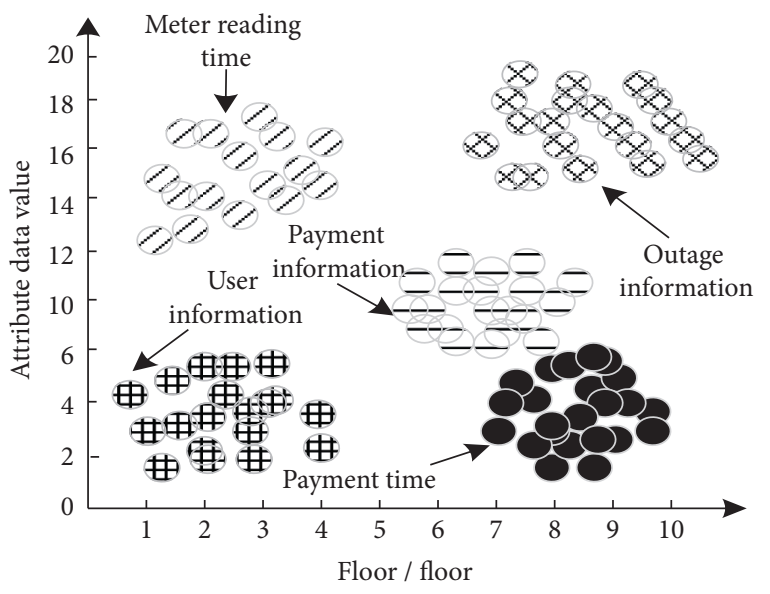

FIgURE 5: Test results of attribute classification.

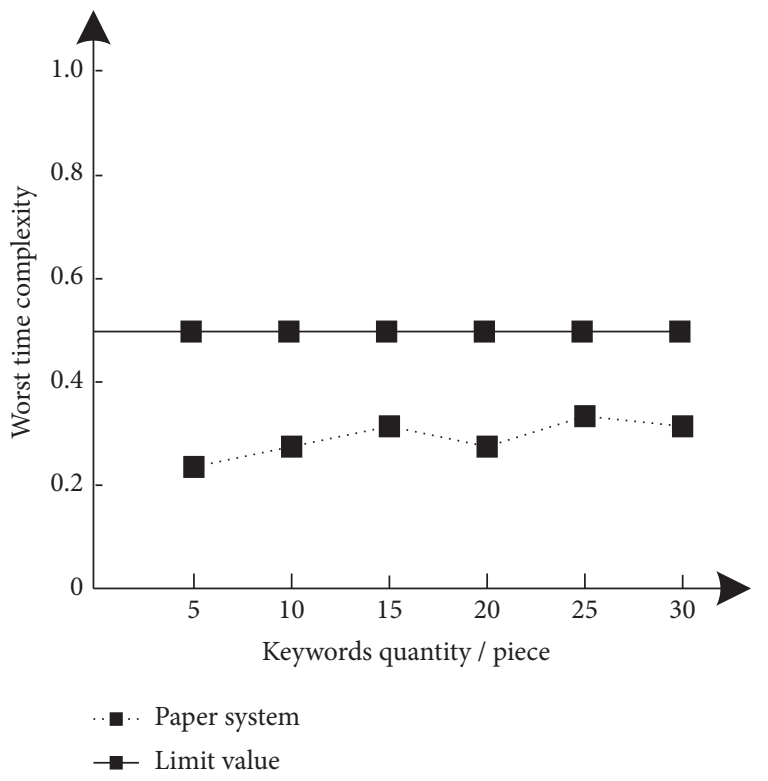

FiguRE 6: Worst time complexity of the system in this research.

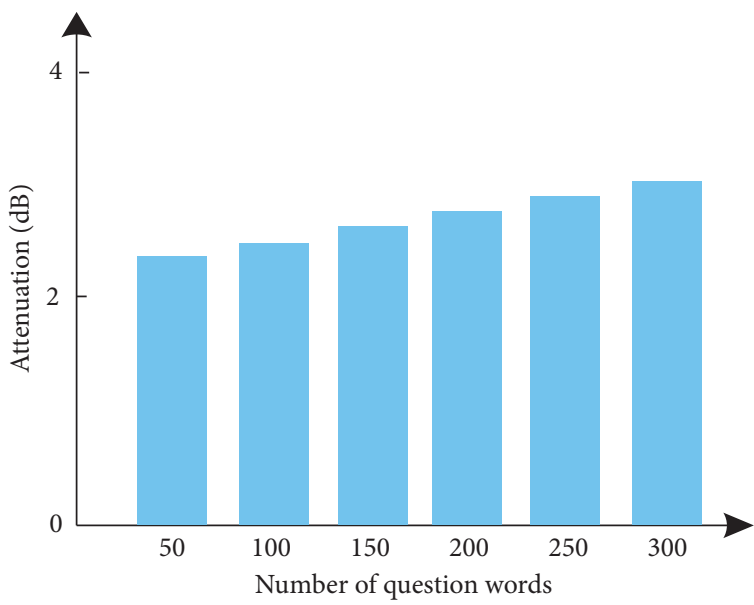

FIGURE 7: Resource scheduling attenuation of the proposed system in this paper. 


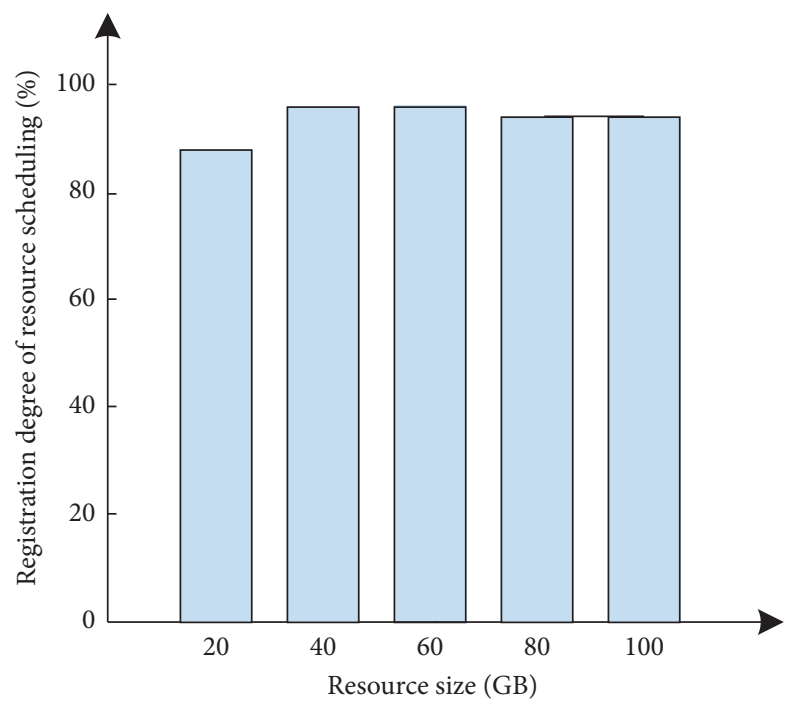

FIGURE 8: Resource scheduling registration degree of the proposed system.

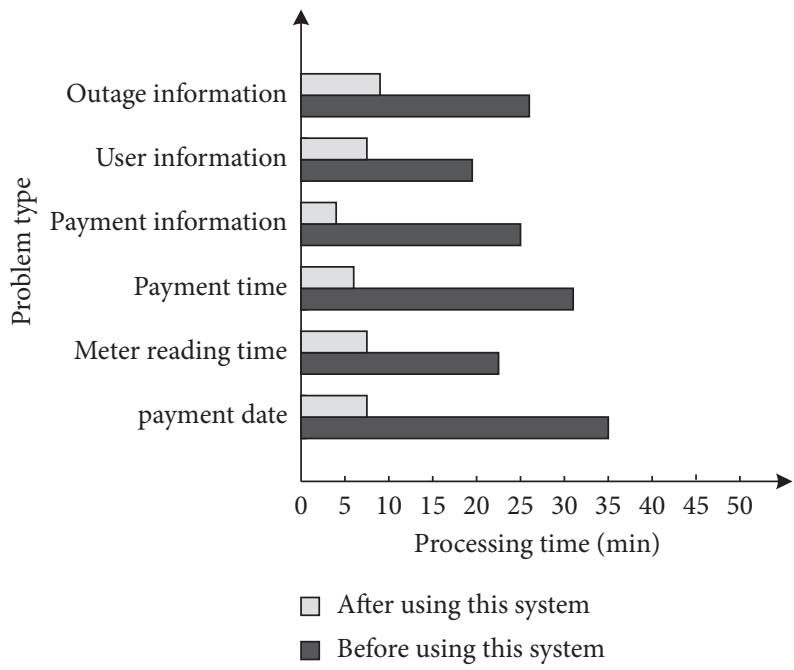

FIgURE 9: Comparison of effects before and after application of the proposed system.

\section{Conclusion}

According to the actual needs of power business customers, taking improving customer service quality as the starting point and improving the operation efficiency of the company as the goal, an intelligent customer service questioning and answering a system for power business scenario based on AI technology is designed through the combination of independent customer service and natural language. When customers ask questions, the solution strategy is obtained by designing the system self-service. The approach first uses the particle swarm optimization algorithm to automatically classify the question attributes and then uses the fuzzy $c$ means clustering algorithm to match the answers with the highest similarity to the questions and return to the customers. The experimental results show that the designed system has low worst-case time complexity, which is up to 0.35 only. The reason is that the system in this paper can use priority information to deal with the problems raised by customers, which is different from the past work where dealing with customers request via priority information is not used.

Compared with past work, the novelty of the proposed approach is shown as follows. The approach is AI-based, which combines the particle swarm optimization algorithm and the fuzzy $c$-means clustering algorithm to automatically classify the question attributes and match the answers with the highest similarity to the questions. It reduces the differences between problem data, eliminates invalid data, and simplifies the data classification process. It enables the proposed system to use priority information to deal with the problems raised by customers, leading to a low worst-case time complexity.

From the perspective of customers, the design system can meet the needs of customers and facilitate customers to obtain the solutions they need in time. From the perspective of the company, the existence of the design system can improve the processing speed of power business, strengthen 
the information construction of the company, ensure its efficiency while saving operation cost, and make the operation and maintenance work of the company smoother. It is a powerful measure to promote the construction of power remote service and can be used for automation of power exchange platform in the future.

\section{Data Availability}

The data used to support the findings of this study are included within the article.

\section{Conflicts of Interest}

The authors declare that they have no conflicts of interest.

\section{References}

[1] O. Bassey, K. L. Butler-Purry, and B. Chen, "Dynamic modeling of sequential service restoration in islanded single master microgrids," IEEE Transactions on Power Systems, vol. 35, p. 1, 2019.

[2] P. S. Challagidad and M. N. Birje, "Local and remote recovery of cloud services using backward atomic backup recovery technique for high availability in strongly consistent cloud service: recovery of cloud service for high availability," International Journal of Advanced Pervasive and Ubiquitous Computing, vol. 11, 2019.

[3] J. Fan, D. F. Wu, and X. X. Liu, "Business data fragmentation and optimization in NB-IoT," Computer Simulation, vol. 37, no. 10, pp. 286-290+296, 2020.

[4] H. Larsen, "Normative power europe or capability-expectations gap? the performativity of concepts in the study of european foreign policy," Journal of Communication and Media Studies: Journal of Common Market Studies, vol. 58, no. 4, 2020.

[5] Y. Qiao, C. Zhang, R. Li, and Z. Liu, "A wireless intelligent business laundry service system," Journal of Computer and Communications, vol. 07, no. 7, pp. 105-113, 2019.

[6] A. Yt, A. Hx, B. Yw, A. Zz, B. Ya, and C. Yx, "Research on knowledge driven intelligent question answering system for electric power customer service," Procedia Computer Science, vol. 187, pp. 347-352, 2021.

[7] Z. Li, X. Liu, W. M. Wang, A. Vatankhah Barenji, G. Q. Huang, and G. Q. Huang, "CKshare: secured cloudbased knowledge-sharing blockchain for injection mold redesign," Enterprise Information Systems, vol. 13, no. 1, pp. 1-33, 2019.

[8] J. Yu, H. Ji, Q. Song, and L. Zhou, "Design and implementation of business access control in new generation power grid dispatching and control system," Procedia Computer Science, vol. 183, no. 22, pp. 761-767, 2021.

[9] Y. Chen, Z. Guo, H. Li, Y. Yang, and Z. Ding, "Selection of a critical time scale of real-time dispatching for power systems with high proportion renewable power sources," IEEE Access, vol. 8, pp. 52257-52267, 2020.

[10] Q. Yao, J. Liu, and Y. Hu, "Optimized active power dispatching strategy considering fatigue load of wind turbines during de-loading operation," IEEE Access, vol. 7, pp. 17439-17449, 2019.

[11] F. Hu, H. Chen, and X. Wang, "An intuitionistic kernel-based fuzzy c-means clustering algorithm with local information for power equipment image segmentation," IEEE Access, vol. 8, pp. 4500-4514, 2020.

[12] C. Liu, Q. Li, and K. Wang, "State-of-charge estimation and remaining useful life prediction of supercapacitors," Renewable and Sustainable Energy Reviews, vol. 150, Article ID 111408, 2021.

[13] Y. Hua, N. Wang, and K. Zhao, "Simultaneous unknown input and state estimation for the linear system with a rankdeficient distribution matrix," Mathematical Problems in Engineering, vol. 2021, Article ID 6693690, 11 pages, 2021.

[14] K. Wang, C. Liu, J. Sun et al., "State of charge estimation of composite energy storage systems with supercapacitors and lithium batteries," Complexity, vol. 2021, Article ID 8816250, 15 pages, 2021.

[15] K. Wang, W. Wang, L. Wang, and L. Li, “An improved SOC control strategy for electric vehicle hybrid energy storage systems," Energies, vol. 13, no. 20, p. 5297, 2020.

[16] X. Feng, Q. li, and K. Wang, "Waste plastic triboelectric nanogenerators using recycled plastic bags for power generation," ACS Applied Materials \& Interfaces, vol. 13, no. 1, pp. 400-410, 2020.

[17] J. Zhao, F. Li, Z. Wang, P. Dong, G. Xia, and K. Wang, "Flexible PVDF nanogenerator-driven motion sensors for human body motion energy tracking and monitoring," Journal of Materials Science: Materials in Electronics, vol. 32, no. 11, pp. 14715-14727, 2021.

[18] S.-I. Gustafsson, "Optimal use of solar collectors for residential buildings," International Journal of Energy Research, vol. 25, no. 11, pp. 993-1004, 2001.

[19] K. Bhattacharjee and M. Pant, "Hybrid particle swarm optimization-genetic algorithm trained multi-layer perceptron for classification of human glioma from molecular brain neoplasia data," Cognitive Systems Research, vol. 58, pp. 173-194, 2019.

[20] J. Too, A. Abdullah, N. Mohd Saad, and W. Tee, "Emg feature selection and classification using a pbest-guide binary particle swarm optimization," Computation, vol. 7, no. 1, 2019.

[21] H. Verma, A. Gupta, and D. Kumar, "A modified intuitionistic fuzzy c-means algorithm incorporating hesitation degree," Pattern Recognition Letters, vol. 122, pp. 45-52, 2019.

[22] P. K. Mishro, S. Agrawal, R. Panda, and A. Abraham, "A novel type-2 fuzzy c-means clustering for brain $\mathrm{mr}$ image segmentation," IEEE Transactions on Cybernetics, no. 99, pp. 1-12, 2020. 